(C)2009 IEEE. Personal use of this material is permitted. However, permission to reprint/republish this material for advertising or promotional purposes or for creating new collective works for resale or redistribution to servers or lists, or to reuse any copyrighted component of this work in other works must be obtained from the IEEE. 
Proceedings of the IEEE

International Conference on Automation and Logistics

Shenyang, China August 2009

\section{Nonlinear Optimal Feedback Control for Lunar Module Soft Landing*}

\author{
Jingyang Zhou and Di Zhou \\ Department of Control Science and \\ Engineering \\ Harbin Institute of Technology \\ Harbin, Heilongjiang Province, China \\ zhouhit@gmail.com
}

\author{
Kok Lay Teo \\ Department of Mathematics and \\ Statistics \\ Curtin University of Technology \\ Perth, WA, Australia \\ K.L.Teo@curtin.edu.au
}

\author{
Guohui Zhao \\ Institute of Mathematical Sciences \\ Dalian University of Technology \\ Dalian, Liaoning Province, China \\ ghzhao6961@hotmail.com
}

\begin{abstract}
In this paper, the optimal control problem to achieve lunar module soft landing with least fuel consumption is considered. The precise three dimensional dynamics is employed to describe the motion of the lunar module. By introducing two new state equations, a closed loop optimal control law is designed with a parameter matrix $K$ to be determined which is the solution of a riccati like differential equation. We present a practical method to calculate the matrix $K$, such that it is avoided to solve the complex riccati like differential equation. Simulation results show the efficiency of the proposed method.
\end{abstract}

Index Terms - Feedback control, Lunar module, Soft landing

\section{INTRODUCTION}

With the development of modern science and technology, many countries begin to explore the moon, the nearest celestial body to the earth. Satellites and probes have been sent out to the moon for investigations. Various research projects are ongoing. Among those missions, landing the lunar rover or astronauts safely on the moon surface is the most challenging one for space scientists.

It is well known that there is no atmosphere around the moon to be used by the lunar module for deceleration, hence lunar soft landing can not be performed in the same way as landing on the earth or mars. The only way to realize soft landing is to use the reverse force thruster which will subsequently consume much of the fuel that the lunar module is carrying. Clearly, if the fuel consumption can be reduced, then more payloads can be equipped. Thus, the optimal control strategy that guarantees the soft landing with least fuel consumption is highly in demand. Consequently, there are now many papers devoted to this area in the literature[1-6]. Meditch discussed the problem of vertical lunar soft landing, where he pointed out that when the thruster works at its maximum force, the mission is equivalent to a time optimal control problem and hence can be solved by existing theory[7]. Xi presented an optimal control law obtained by utilizing Pontryagin Maximum Principle for the soft landing of a lunar module. Here, it is assumed that some of the control variables are not bounded[8]. Wang proposed a suboptimal guidance law for lunar soft landing with the assumption of a uniform gravity field on the moon surface. The guidance law is a function of time-to-go[9]. Liu designed an optimal control strategy for the soft landing of a lunar module with a pre- selected terminal time by using the control parameterization and a time scaling transform[10]. Among those published research articles, the dynamical system considered in most of these articles is a two dimensional dynamics. The descent trajectory of the lunar module is assumed to remain in a vertical plane without consideration of the lateral movement. Neither the influence of the moon rotation is taken into account. To be realistic, a three dimensional dynamics taking into consideration of moon rotation is thus employed to describe the motion of the lunar module in this paper[11].

Most existed research works treat the mission of lunar soft landing as an open loop optimal control problem, and hence open loop optimal or suboptimal control laws are designed to realize the soft landing. In this paper, by transformation of the precise three dimensional dynamics, we propose a closed loop optimal control law for lunar module soft landing. A practical computational method is also presented to solve the parameter matrix $\boldsymbol{K}$, which is the solution of a riccati like differential equation. Such that, calculation of the complex riccati like differential equation is avoided.

\section{PROBLEM FORMULATION}

Lunar module soft landing starts from the parking orbit of the moon, after Hohmann transfer the module enters an elliptical orbit with the aposelene and perilune, which are, respectively, $110 \mathrm{~km}$ and $15 \mathrm{~km}$ distance from the moon surface. The reverse force thruster begins to work from the perilune to decelerate the initial velocity of the module. With the cooperation of the attitude control thrusters, the module is guided to reach the landing target with a small and safe finial velocity. In this paper, we study the closed loop optimal guidance scheme for ensuring the soft landing of the lunar module from the perilune to the moon surface.

The motion of the lunar module soft landing can be described by a set of three dimensional coordinates (Fig.1). Suppose oxyz and $o x_{L} y_{L} z_{L}$ are, respectively, the Lunar Central Inertial Coordinate and Lunar Fixed Coordinate with the moon equator as the reference plane. $A x_{1} y_{1} z_{1}$ is orbit coordinate, $A$ is the position of the lunar module. All the three coordinates are right handed systems. $\alpha$ and $\beta$ represent rotation angles between oxyz and $A x_{1} y_{1} z_{1}$. The

\footnotetext{
* This work is partially supported by NSFC Grant \#60535010 to Q.-H. Meng.
} 
direction of the thrust force $P$ in the coordinate $A x_{1} y_{1} z_{1}$ can be described by $\vartheta$ and $\psi \cdot \gamma$ is the rotation angle between $o x y z$ and $o x_{L} y_{L} z_{L}$. Without lose of generality, we assume that $o x y z$ and $o x_{L} y_{L} z_{L}$ coincide with each other at the beginning of soft landing. Based on Newton's second law, system dynamic equations can be derived [11] to give

$$
\left\{\begin{array}{l}
\dot{x}_{L}=V_{x L} \\
\dot{y}_{L}=V_{y L} \\
\dot{z}_{L}=V_{z L} \\
\dot{V}_{x L}=B \frac{Q V_{r}}{m}+g_{x L}-2 \omega_{L} V_{z L} \\
\dot{V}_{y L}=C \frac{Q V_{r}}{m}+g_{y L} \\
\dot{V}_{z L}=D \frac{Q V_{r}}{m}+g_{z L}+2 \omega_{L} V_{x L} \\
\dot{m}=-Q
\end{array}\right.
$$

where

$$
\begin{aligned}
B= & (\cos \alpha \cos \beta \cos \gamma-\sin \alpha \sin \gamma) \sin \vartheta \cos \psi \\
& -(\sin \alpha \cos \beta \cos \gamma+\cos \alpha \sin \gamma) \sin \vartheta \sin \psi \\
& +\sin \beta \cos \gamma \cos \vartheta \\
C= & -\cos \alpha \sin \beta \sin \vartheta \cos \psi+\cos \beta \cos \vartheta \\
& +\sin \alpha \sin \beta \sin \vartheta \sin \psi \\
D= & (\cos \alpha \cos \beta \sin \gamma+\sin \alpha \cos \gamma) \sin \vartheta \cos \psi \\
& -(\sin \alpha \cos \beta \sin \gamma-\cos \alpha \cos \gamma) \sin \vartheta \sin \psi \\
& +\sin \beta \sin \gamma \cos \vartheta
\end{aligned}
$$

$x_{L}, y_{L}, z_{L}$ and $V_{x L}, V_{y L}, V_{z L}$ are the positions and velocities in the Lunar Fixed Coordinate. $m$ is the mass of the lunar module. $Q$ and $V_{r}$ represent the fuel consumption rate and the specific impulse of the thruster. $g_{x L}, g_{y L}$ and $g_{z L}$ denote the components of lunar gravity in $o x_{L} y_{L} z_{L} \cdot \omega_{L}$ is the angular velocity of the moon rotation.

By introducing two new state equations

$$
\begin{aligned}
\dot{\vartheta} & =v \\
\dot{\psi} & =w
\end{aligned}
$$

and letting
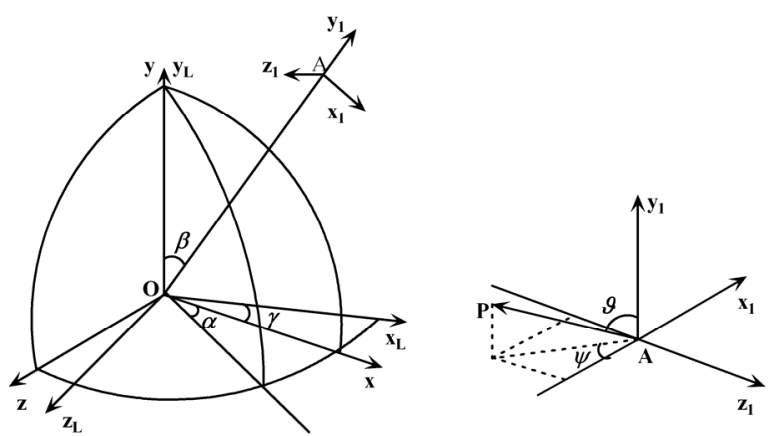

Fig. 1 Coordinate systems

$$
\begin{aligned}
\boldsymbol{x} & =\left[x_{L}, y_{L}, z_{L}, V_{x L}, V_{y L}, V_{z L}, \vartheta, \psi, m\right]^{T} \\
& =\left[x_{1}, x_{2}, x_{3}, x_{4}, x_{5}, x_{6}, x_{7}, x_{8}, x_{9}\right]^{T}
\end{aligned}
$$

$$
\boldsymbol{u}=[Q, v, w]^{T}=\left[u_{1}, u_{2}, u_{3}\right]^{T}
$$

The original system dynamics (1) can be rewritten in the form of an affine nonlinear system given below.

$$
\dot{\boldsymbol{x}}(t)=\boldsymbol{f}(\boldsymbol{x}(t))+\boldsymbol{B}(\boldsymbol{x}(t)) \boldsymbol{u}(t)
$$

where

$$
\begin{aligned}
& \boldsymbol{f}(\boldsymbol{x})=\left[x_{4}, x_{5}, x_{6}, g_{x L}-2 \omega_{L} x_{6}, g_{y L}, g_{z L}+2 \omega_{L} x_{4}, 0,0,0\right]^{T} \\
& \boldsymbol{B}(\boldsymbol{x})=\left[\begin{array}{ccccccccc}
0 & 0 & 0 & M_{1} & M_{2} & M_{3} & 0 & 0 & -1 \\
0 & 0 & 0 & 0 & 0 & 0 & 1 & 0 & 0 \\
0 & 0 & 0 & 0 & 0 & 0 & 0 & 1 & 0
\end{array}\right]^{T} \\
& M_{1}=\left[(\cos \alpha \cos \beta \cos \gamma-\sin \alpha \sin \gamma) \sin x_{7} \cos x_{8}\right. \\
& -(\sin \alpha \cos \beta \cos \gamma+\cos \alpha \sin \gamma) \sin x_{7} \sin x_{8} \\
& \left.+\sin \beta \cos \gamma \cos x_{7}\right] \frac{V_{r}}{x_{9}} \\
& M_{2}=\left[-\cos \alpha \sin \beta \sin x_{7} \cos x_{8}+\sin \alpha \sin \beta \sin x_{7} \sin x_{8}\right. \\
& \left.+\cos \beta \cos x_{7}\right] \frac{V_{r}}{x_{9}} \\
& M_{3}=\left[(\cos \alpha \cos \beta \sin \gamma+\sin \alpha \cos \gamma) \sin x_{7} \cos x_{8}\right. \\
& -(\sin \alpha \cos \beta \sin \gamma-\cos \alpha \cos \gamma) \sin x_{7} \sin x_{8} \\
& \left.+\sin \beta \sin \gamma \cos x_{7}\right] \frac{V_{r}}{x_{9}}
\end{aligned}
$$

The control vector $\boldsymbol{u}$ is bounded satisfying:

$$
\boldsymbol{u}_{\text {min }} \leq \boldsymbol{u}(t) \leq \boldsymbol{u}_{\text {max }}, \forall t \geq 0
$$

The initial conditions of the soft landing are determined by the states of the lunar module at perilune with initial time $t_{0}=0$. The terminal conditions are specified by the requirements of the soft landing, i.e., when the lunar module reaches the target, its velocity should approach to zero while the terminal time $t_{f}$ is free. So the initial and terminal conditions can be expressed as:

$$
\begin{gathered}
\boldsymbol{x}\left(t_{0}\right)=\left[x_{L 0}, y_{L 0}, z_{L 0}, V_{x L 0}, V_{y L 0}, V_{z L 0}, \vartheta_{0}, \psi_{0}, m_{0}\right]^{T} \\
\boldsymbol{\Phi}=\left[\begin{array}{c}
x_{L}\left(t_{f}\right)-x_{L r} \\
y_{L}\left(t_{f}\right)-y_{L r} \\
z_{L}\left(t_{f}\right)-z_{L r} \\
V_{x L}\left(t_{f}\right)-0 \\
V_{y L}\left(t_{f}\right)-0 \\
V_{z L}\left(t_{f}\right)-0
\end{array}\right]=0
\end{gathered}
$$

respectively, where $\left(x_{L r}, y_{L r}, z_{L r}\right)$ represents the position of the landing target in the Lunar Fixed Coordinate. Our aim is to design a close loop optimal control law such that the lunar module achieves the task of soft landing with the least fuel consumption as well as the minimum flying time. The cost function can be formulated as follow

$$
J=m_{0}-m_{t_{f}}+t_{f}
$$

We may now formally state our optimal control problem as follows.

Problem (P): Given system (4), find a closed loop optimal control vector $\boldsymbol{u}$ such that the cost function (10) is 
minimized subject to the control constraint (7), the initial condition (8) and the terminal condition (9).

\section{Closed Loop Optimal Control}

To design the closed loop optimal control law, we assume that the following condition is satisfied throughout.

Assumption (A1): $\boldsymbol{f}(\boldsymbol{x}(t)) \neq 0$ for all $t \in[0, T]$.

It can be seen easily from (5) that $\mathbf{A 1}$ is always satisfied in our problem.

Theorem 1[12]: For the system (4) and the cost function

$$
J(\boldsymbol{u})=\Phi_{0}(\boldsymbol{x}(T))+\int_{0}^{T}(\boldsymbol{x}-\boldsymbol{c})^{T} \boldsymbol{Q}(\boldsymbol{x}-\boldsymbol{c})+\boldsymbol{u}^{T} \boldsymbol{R} \boldsymbol{u} d t
$$

where $\boldsymbol{x} \in R^{n}, \boldsymbol{u} \in R^{r}, \boldsymbol{Q}$ and $\boldsymbol{R}$ are respectively symmetric positive semi-definite and symmetric positive matrices with appropriate dimensions, $\boldsymbol{c}$ is a constant vector.

The closed loop optimal controller $\boldsymbol{u}^{*}$ which could minimise the cost function (11) can be expressed as

$$
\boldsymbol{u}^{*}=\frac{1}{2} \boldsymbol{R}^{-1}\left[\boldsymbol{B}\left(\boldsymbol{x}^{*}\right)\right]^{T} \boldsymbol{K}(t) \boldsymbol{f}\left(\boldsymbol{x}^{*}\right)
$$

$\boldsymbol{x}^{*}$ is the optimal states under $\boldsymbol{u}^{*} . \boldsymbol{K}(t)$ is the solution of the following riccati like differential equation

$$
\dot{\boldsymbol{K}}+\boldsymbol{K} \boldsymbol{F}+\boldsymbol{F}^{T} \boldsymbol{K}+\boldsymbol{D}^{T} \boldsymbol{K}+\frac{1}{2} \boldsymbol{K} \boldsymbol{F B R} \boldsymbol{R}^{-1} B^{T} \boldsymbol{K}-2 \boldsymbol{Q}(\boldsymbol{x}-\boldsymbol{c}) \frac{\boldsymbol{f}^{T}}{\boldsymbol{f}^{T} \boldsymbol{f}}=0
$$

where $\boldsymbol{F}=\partial \boldsymbol{f} / \partial \boldsymbol{x}, \boldsymbol{D}=\partial(\boldsymbol{B} \boldsymbol{u}) / \partial \boldsymbol{x}$, with the terminal condition

$$
\boldsymbol{K}(T) \boldsymbol{f}(\boldsymbol{x}(T))=\frac{\partial \Phi_{0}(\boldsymbol{x}(T))}{\partial \boldsymbol{x}(T)}
$$

$\boldsymbol{u}^{*}$ satisfies the necessary conditions for optimality.

As $\boldsymbol{B}$ and $\boldsymbol{f}$ are functions of system states that are already known, so to realize the optimal control we only need to calculate the parameter matrix $\boldsymbol{K}$. It is noticed that $\boldsymbol{K}$ is related with $\boldsymbol{x}^{*}$. This give rise to a complex two-point boundary value problem(TPBVP). When the system level is high, it is a very time consuming task.

In the next section, with the help of optimal control software MISER3.3[13], we present a practical method to calculate $\boldsymbol{K}$ without solving this TPBVP directly.

\section{A Practical Computational Method}

It is well known that MISER3.3 is efficient computational software based on the theory of control parameterization[14] which could solve the problems of open loop optimal control. Standard MISER3.3 could deal with the problem whose control takes the form of piecewise constant or piecewise linear. To satisfy the requirements of our problem, we made some modifications to make MISER3.3 deal with continuous control problems. So, by using the modified MISER3.3, we can obtain the open loop optimal solution of problem (P) as $\left(\tilde{\boldsymbol{u}}, \tilde{\boldsymbol{x}}, t_{f}\right), \tilde{\boldsymbol{u}}$ is the open loop optimal control and $\tilde{\boldsymbol{x}}$ denotes the optimal states under $\tilde{\boldsymbol{u}}, t_{f}$ is the optimal terminal time. Letting

$$
J(\boldsymbol{K})=\int_{0}^{t_{f}}(\tilde{\boldsymbol{u}}-\boldsymbol{u})^{2} d t
$$

where

$$
\boldsymbol{u}=\boldsymbol{R}^{-1}[\boldsymbol{B}(\tilde{\boldsymbol{x}})]^{T} \boldsymbol{K}(t) \boldsymbol{f}(\tilde{\boldsymbol{x}}) / 2
$$

Then if $\boldsymbol{u} \rightarrow \tilde{\boldsymbol{u}}$, there must be $J(\boldsymbol{K}) \rightarrow 0$. Now our aim is to find the matrix $\boldsymbol{K}(t)$ which could minimise $J(\boldsymbol{K})$, therefore to minimise $J$.

It is noticed that $\boldsymbol{K}(t)$ is the solution of the riccati like differential equation (13), which is continuously throughout $\left[\begin{array}{ll}0 & t_{f}\end{array}\right]$. For this reason, we may use cubic splines as basis functions to approximate the elements of $\boldsymbol{K}(t)$. Letting

$$
[\boldsymbol{K}(t)]_{i, j} \approx \sum_{k=-1}^{n_{p}+1}\left(c_{i, j, k}\right) \Omega\left(\left(\frac{t_{f}}{n_{p}}\right) t-k\right)
$$

where $c_{i, j, k}$ are real constant parameters that should be determined, $i, j \in\{1,2, \ldots, n\}, k \in\left\{-1,0,1,2, \ldots, n_{p}+1\right\} . n_{p}$ is the number of equality subintervals on $\left[0 t_{f}\right], n_{p}+3$ is the total number of cubic spline basis functions used in the approximation for each $[\boldsymbol{K}(t)]_{i, j}$. Here we use the cubic spline function $\Omega(s)$ defined by

$$
\Omega(s)=\left\{\begin{array}{cc}
0 & |s|>2 \\
-\frac{1}{6}|s|^{3}+s^{2}-2|s|+\frac{4}{3} & 1 \leq|s| \leq 2 \\
\frac{1}{2}|s|^{3}-s^{2}+\frac{2}{3} & |s|<1
\end{array}\right.
$$

as our basis functions.

Now the original optimal control problem is transformed in to an optimal parameter selection problem $\left(\mathrm{P}_{\mathrm{c}}\right)$ as follow:

Problem $\left(\mathrm{P}_{\mathrm{c}}\right)$ : On the time interval $\left[0 t_{f}\right]$, for equations (14)-(17), finding a suitable combination of parameters $c_{i, j, k}$, which would make $\boldsymbol{u} \rightarrow \tilde{\boldsymbol{u}}$ and minimise $J(\boldsymbol{K})$.

To solve problem $\left(\mathrm{P}_{\mathrm{c}}\right)$, we could simply let

$$
\boldsymbol{C}=\frac{\partial J(\boldsymbol{K})}{\partial c_{i, j, k}}=\int_{0}^{T} \frac{\partial(\tilde{\boldsymbol{u}}-\boldsymbol{u})^{2}}{\partial c_{i, j, k}} d t=0
$$

which represents a linear equation system. By solving (18), we can obtain the optimal solution of problem $\left(\mathrm{P}_{\mathrm{c}}\right)$.

Examining (15), we observe that $\boldsymbol{K}(t)$ appears with $[\boldsymbol{B}(\tilde{\boldsymbol{x}})]^{T}$ multiplied from the left, so the columns of $[\boldsymbol{B}(\tilde{\boldsymbol{x}})]^{T}$ whose elements are all zeros do not affect $[\boldsymbol{B}(\tilde{\boldsymbol{x}})]^{T} \boldsymbol{K}(t)$. Hence, there is no need to calculate those $c_{i, j, k}$ corresponding to the zero columns. The computational effort is thus reduced considerably.

\section{NUMERICAL SimULATIONS}

The initial conditions of the lunar module are given as: $x_{L 0}=819.371 \mathrm{~km}, y_{L 0}=1428.867 \mathrm{~km}, z_{L 0}=599.6306 \mathrm{~km}$, $V_{x L 0}=1115 \mathrm{~m} / \mathrm{s}, V_{y L 0}=-981.82 \mathrm{~m} / \mathrm{s}, V_{z L 0}=816 \mathrm{~m} / \mathrm{s}, m_{0}=600$ $\mathrm{kg}$. At the beginning time of the soft landing, rotation angle 
$\gamma\left(t_{0}\right)=0^{\circ}$. Specific impulse $V_{r}=300 \times 9.8 \mathrm{~m} / \mathrm{s}$ and angular velocity of the moon rotation $\omega_{L}=2.661699 \times 10^{-6} \mathrm{rad} / \mathrm{s}$. The landing target is in Mare Imbrium on the moon surface with $38.3^{\circ}$ North latitude and $35^{\circ}$ West longitude. When the module reaches the moon surface, the terminal velocity with respect to the moon should be less than $3 \mathrm{~m} / \mathrm{s}$.

From the open loop optimal solutions obtained by MISER3.3, we know that the reverse force thruster works with its maximum thrust force all the time. Therefore we could simply let $u_{1}=0.51 \mathrm{~kg} / \mathrm{s}$ which guarantees that the thrust force $P=1500 \mathrm{~N}$. So we do not need to calculate those $c_{i, j, k}$ corresponding to $u_{1}$. By examining (5) and (15), we notice that the last three elements of $\boldsymbol{f}(\boldsymbol{x})$ are zeros and $\boldsymbol{K}(t)$ is multiplied with $\boldsymbol{f}(\boldsymbol{x})$ from the right. So the last three columns of $\boldsymbol{K}(t)$ could not affect $\boldsymbol{K}(t) \boldsymbol{f}(\boldsymbol{x})$. Calculation for the corresponding $c_{i, j, k}$ is also avoided. To reduce the computational effort, we may simply set the inactive components of $\boldsymbol{K}(t)$ to zero. In our problem, we only need to calculate 12 elements of $\boldsymbol{K}(t)$ corresponding to $u_{2}$ and $u_{3}$, i.e., $[\boldsymbol{K}(t)]_{i, j}, i \in\{7,8\}, j \in\{1,2, \ldots, 6\}$.

The time history of simulation is partitioned equally into thirty subintervals. The open loop optimal solutions are obtained by MISER3.3 as $\left(\tilde{\boldsymbol{u}}, \tilde{\boldsymbol{x}}, t_{f}\right)$. Substituting $\left(\tilde{\boldsymbol{u}}, \tilde{\boldsymbol{x}}, t_{f}\right)$ into (18), then the linear equations system can be solved by Matlab and the optimal parameters $c_{i, j, k}$ are got. Weighting matrix $\boldsymbol{R}$ can be chosen as $\boldsymbol{R}=\operatorname{diag}(1,1,1)$. The closed loop optimal control law (15) is then obtained which could guarantees the soft landing of lunar module.

Under the closed loop optimal control, the terminal conditions of the module are $x_{L}\left(t_{f}\right)=1117.2911 \mathrm{~km}$, $y_{L}\left(t_{f}\right)=1077.1753 \mathrm{~km}, z_{L}\left(t_{f}\right)=782.304 \mathrm{~km}, V_{x L}\left(t_{f}\right)=0.686$ $\mathrm{m} / \mathrm{s}, V_{x L}\left(t_{f}\right)=-1.08 \mathrm{~m} / \mathrm{s}, V_{x L}\left(t_{f}\right)=0.217 \mathrm{~m} / \mathrm{s}$.

Simulation results are shown by Fig. 2-8. Fig. 2-4 are the time histories of control outputs. It can be seen that the thruster works with its maximum thrust force, the closed loop angular velocity control laws coincide with the open loop ones precisely. Under the closed loop optimal control, lunar module lands on the moon surface after 542.241s, all the velocities along three directions approach to zero(Fig.5-7), terminal velocity of the module with respect to the moon is $1.297 \mathrm{~m} / \mathrm{s}$. The distance between the lunar module and the preselected landing target is $25.6 \mathrm{~m}$. Optimal descent trajectory is shown in Fig. 8 .

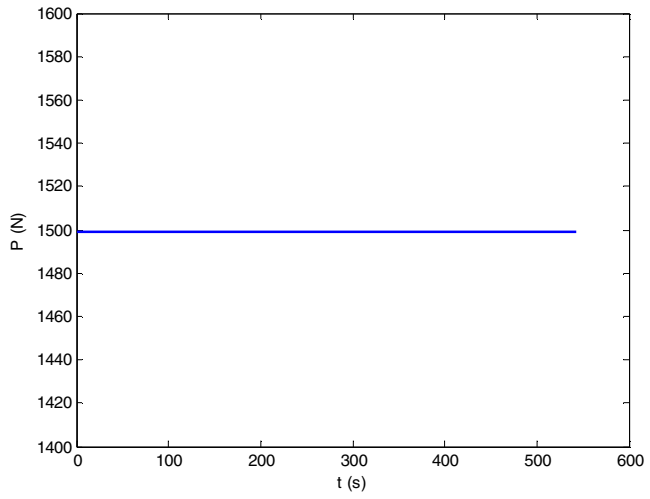

Fig. 2 Thrust force $\mathrm{P}$

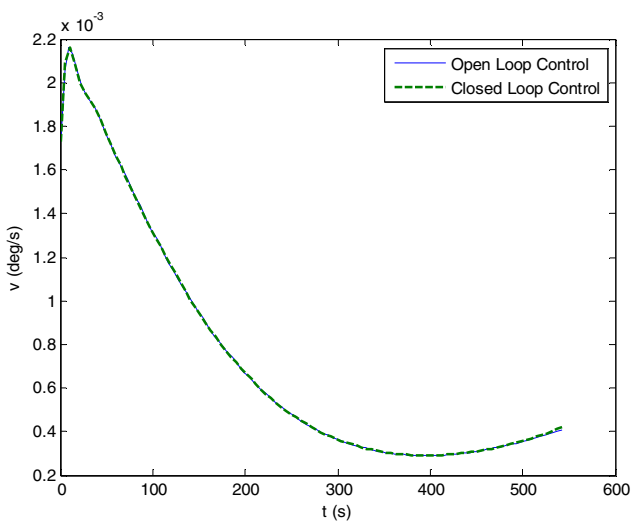

Fig. 3 Angular velocity control v

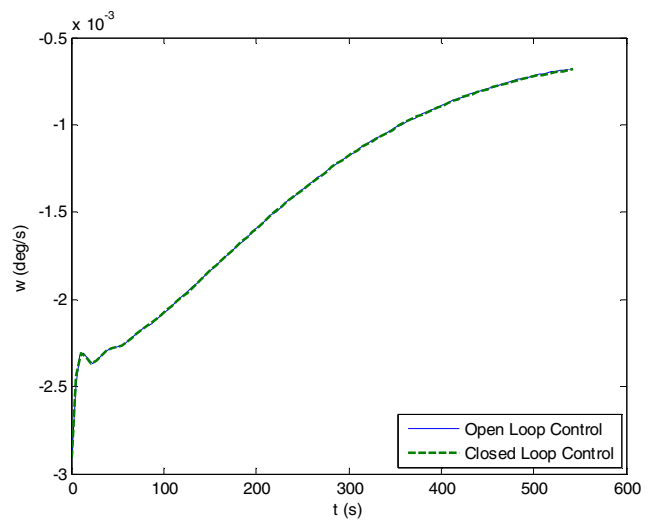

Fig. 4 Angular velocity control w

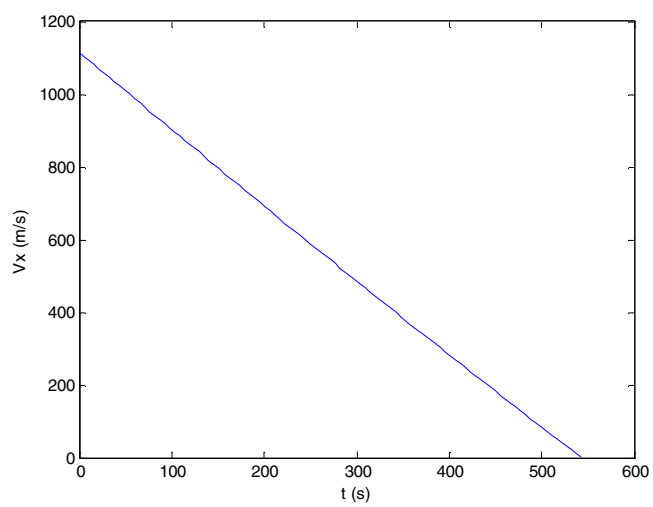

Fig. 5 Velocity along $\mathrm{x}$ axis 


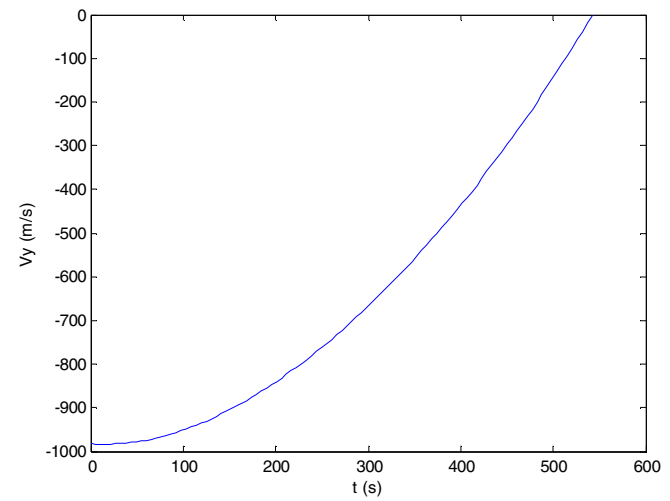

Fig. 6 Velocity along y axis

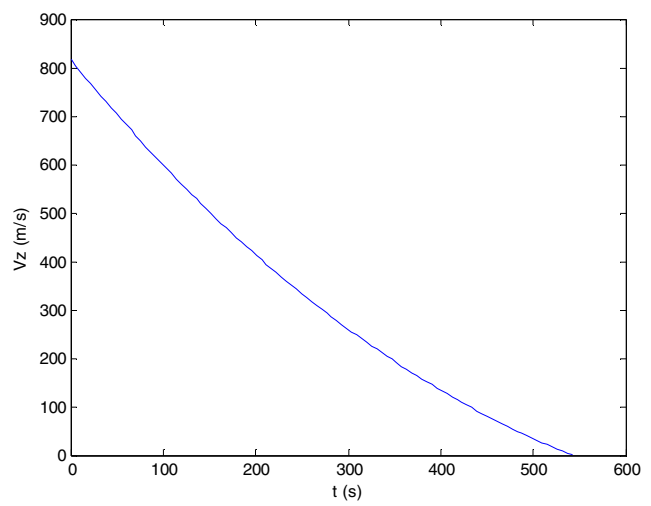

Fig. 7 Velocity along $\mathrm{z}$ axis

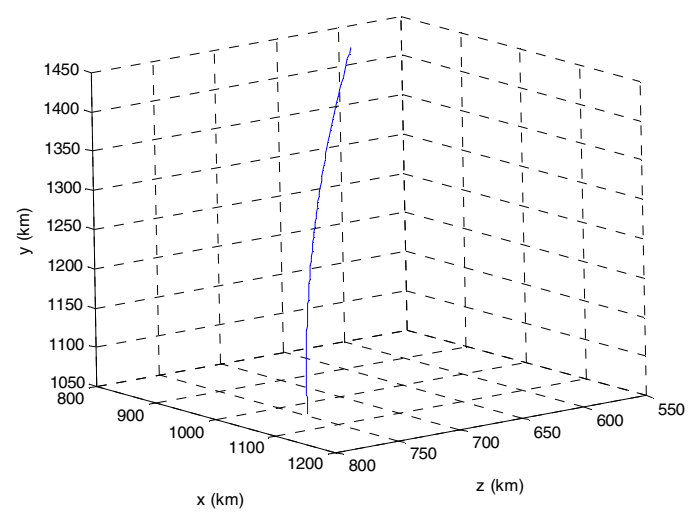

Fig. 8 Optimal descent trajectory

\section{CONCLUSIONS}

For the optimal control problem of lunar module soft landing, based on the precise three dimensional dynamics, a closed loop optimal control law is presented. To realize the optimal control law, we propose a practical method to calculate the parameter matrix $\boldsymbol{K}$, and it is avoided to solve the complex riccati like differential equations. Computational effort is reduced substantially. Performance of the proposed method is verified by simulations.

\section{REFERENCES}

[1] K.M. Ma, L.J Chen, and Z.C. Wang, "Practical design of control law for flight vehicle soft landing," Missiles and Space Vehicles, no. 2, pp.39-43, 2001.

[2] X.G. Ruan, "A nonlinear nuerocontrol scheme for lunar soft landing," Journal of Astronautics, vol.19, no.1, pp.35-43, 1998.

[3] S.R. Hebertt, "Soft landing on a planet: a trajectory planning approach for the liouvillian model," Proceedings of American Control Conference, pp.2936-2940, 1999.

[4] D.Y. Wang, T.S. Li, and X.R. Ma, "Numerical solution of TPBVP in optimal lunar soft landing," Aerospace Control, no. 3, pp.44-49, 2000.

[5] M. Xu, and J.F. Li, "Optimal control of lunar soft landing," Journal of Tsinghua University (Science and Technology), vol. 41, no. 8, pp.87-89, 2001.

[6] Z. Wang, J.F. Li, N.G. Cui and T. Liu., "Genetic algorithm optimization of lunar probe soft landing trajectories," Journal of Tsinghua University (Science and Technology), vol. 43, no. 8, pp.1056-1059, 2003.

[7] S.J. Meditch, "On the problem of optimal thrust programming for a lunar soft landing," IEEE Transaction on Automatic Control, vol. 9, no. 4, pp. 477-484, 1964.

[8] X.N. Xi, G.Q. Zeng, X. Ren, and H.Y. Zhao, Orbit design of lunar probe, $1^{\text {st }}$ ed., National Defence Industry Press, 2001, pp.143-165.

[9] D.Y. Wang, T.S. Li, H. Yan, and X.R. Ma, "A suboptimal fuel guidance law for lunar soft landing," Journal of Astronautics, vol. 21, no. 4, pp. 55$63,2000$.

[10]X.L. Liu, G.R. Duan, and K.L. Teo, "Optimal soft landing control for moon lander," Automatica, vol. 44, no. 4, pp.1097-1103, 2008.

[11]J.Y. Zhou, and D. Zhou, "Precise modeling and optimal orbit design of lunar modules soft landing," Journal of Astronautics, vol. 28, no. 6, pp. 1462-1466, 2007.

[12]H. Wing, and J. Lee. "Computational studies of optimal controls," pp. 94113,1996

[13]L.S. Jennings, M.E. Fisher, K.L. Teo, and C.J. Goh, "MISER3: Solving optimal control problems-an update," Advance Engineering Software, vol. 13, no. 4, pp. 190-196, 1991.

[14]K.L. Teo, C.J. Goh, and K.H. Wong, A unified computational approach to optimal control problems, $1^{\text {st }}$ ed., John Wiley \& Sons Press, 1991, pp. 99161 . 